PROCEEDINGS OF THE

AMERICAN MATHEMATICAL SOCIETY

Volume 129, Number 8, Pages 2419-2430

S 0002-9939(01)05943-3

Article electronically published on January 18, 2001

\title{
EINSTEIN MANIFOLDS AND CONTACT GEOMETRY
}

\author{
CHARLES P. BOYER AND KRZYSZTOF GALICKI
}

(Communicated by Christopher Croke)

\begin{abstract}
We show that every K-contact Einstein manifold is Sasakian-
Einstein and discuss several corollaries of this result.
\end{abstract}

\section{INTRODUCTION}

Recently the authors and their collaborators (cf. [BG1, BG2]) have used the geometry of special types of Riemannian contact manifolds to construct Einstein metrics of positive scalar curvature. In particular, in BG2 we studied SasakianEinstein geometry. Since Sasakian geometry is the odd dimensional analogue of Kähler geometry, one might inquire as to the validity of an odd dimensional Goldberg Conjecture. Recall that the Goldberg conjecture Gol] states that a compact almost Kähler manifold that is also Einstein is Kähler-Einstein, that is, the almost complex structure is integrable. This conjecture has been confirmed by Sekigawa Sek1, Sek2 in the case of nonnegative scalar curvature. Since Sasakian-Einstein metrics necessarily have positive scalar curvature, it is tempting to believe that an odd dimensional Goldberg Conjecture holds true. The form in which one would expect this conjecture to be true assumes that the metric be bundle-like. If the Reeb vector field is quasi-regular so that under a compactness assumption there is an orbifold fibration over an almost Kähler-Einstein orbifold it seems quite likely that such a result should follow directly from Sekigawa's result. However, in general one does not have such an orbifold submersion. We handle this more general case by considering the closures of the leaves of the characteristic foliation together with a construction of Molino [Mol1, Mol2] which in the presence of a bundle-like Riemannian metric gives the existence of a sheaf of commuting Killing vector fields. This sheaf can then be used to approximate the geometry of the general case by orbifold submersions. Thus the main purpose of this note is to prove the following:

Theorem A. Let $(M, \eta, g)$ be a compact metric contact manifold whose Riemannian metric $g$ is bundle-like with respect to the characteristic foliation on $M$. Then if $g$ is Einstein, then it is Sasakian-Einstein. Equivalently, every compact K-contact Einstein manifold is Sasakian-Einstein.

We also discuss some consequences of Theorem A to almost Kähler structures on cones, and to some related work on $\eta$-Einstein manifolds.

Received by the editors December 16, 1999.

2000 Mathematics Subject Classification. Primary 53C25.

This work was partially supported by NSF grant DMS-9970904.

(C)2001 American Mathematical Society 


\section{Some metric CONTACT GEOMEtry}

Let $(M, \mathcal{D})$ be a contact manifold and fix a contact 1 -form $\eta$ such that $\mathcal{D}=$ ker $\eta$. The pair $(\mathcal{D}, \omega)$, where $\omega$ is the restriction of $d \eta$ to $\mathcal{D}$ gives $\mathcal{D}$ the structure of a symplectic vector bundle. We denote by $\mathcal{J}(\mathcal{D})$ the space of all almost complex structures $J$ on $\mathcal{D}$ that are compatible with $\omega$, that is the subspace of smooth sections $J$ of the endomorphism bundle End $\mathcal{D}$ that satisfy

$$
J^{2}=-\mathbb{I}, \quad d \eta(J X, J Y)=d \eta(X, Y), \quad d \eta(X, J X)>0
$$

for any smooth sections $X, Y$ of $\mathcal{D}$. Notice that each $J \in \mathcal{J}(\mathcal{D})$ defines a Riemannian metric $g_{\mathcal{D}}$ on $\mathcal{D}$ by setting $g_{\mathcal{D}}(X, Y)=d \eta(X, J Y)$. One easily checks that $g_{\mathcal{D}}$ satisfies the compatibility condition $g_{\mathcal{D}}(J X, J Y)=g_{\mathcal{D}}(X, Y)$. Furthermore, the map $J \mapsto g_{\mathcal{D}}$ is one-to-one, and the space $\mathcal{J}(\mathcal{D})$ is contractible. A choice of $J$ gives $M$ an almost CR structure with a strictly pseudoconvex Levy form.

Moreover, by extending $J$ to all of $T M$ one obtains an almost contact structure [Bl], YK]. There are some choices of conventions to make here. We define the section $\Phi$ of End $T M$ by $\Phi=J$ on $\mathcal{D}$ and $\Phi \xi=0$, where $\xi$ is the Reeb vector field associated to $\eta$. We can also extend the transverse metric $g_{\mathcal{D}}$ to a metric $g$ on all of $M$ by

$$
g(X, Y)=g_{\mathcal{D}}+\eta(X) \eta(Y)=d \eta(X, \Phi Y)+\eta(X) \eta(Y)
$$

for all vector fields $X, Y$ on $M$. One easily sees that $g$ satisfies the compatibility condition $g(\Phi X, \Phi Y)=g(X, Y)-\eta(X) \eta(Y)$. A contact manifold $M$ with a fixed contact form $\eta$ together with a vector field $\xi$, a section $\Phi$ of End $T M$, and a Riemannian metric $g$ which satisfy the conditions

$$
\eta(\xi)=1, \quad \Phi^{2}=-\mathbb{I}+\xi \otimes \eta, \quad g(\Phi X, \Phi Y)=g(X, Y)-\eta(X) \eta(Y)
$$

is known [B] as a metric contact structure on $M$.

Let us consider the characteristic foliation $\mathcal{F}_{\xi}$ generated by the Reeb vector field $\xi$. If $\mathcal{F}_{\xi}$ is a Riemannian foliation [Rei2, Mol1, that is, the holonomy pseudogroup induces isometries of Riemannian metrics on the local leaf spaces of the local submersions defining $\mathcal{F}_{\xi}$, then by pulling back the metrics on the local leaf spaces one obtains a transverse metric $g_{\mathcal{D}}$ on the vector bundle $\mathcal{D}$ that is invariant under the (Reeb) flow generated by the Reeb vector field $\xi$. This is equivalent to the metric $g$ on $M$ given by (2.2) being bundle-like Rei1, Rei2. Hence

Definition 2.4. A contact metric manifold $(M, \eta, g)$ is said to be bundle-like if the Riemannian metric $g$ is bundle-like.

We have

Proposition 2.5. On a complete contact metric manifold $(M, \eta, g)$, the following are equivalent:

(1) $g$ is bundle-like.

(2) The Reeb flow is an isometry.

(3) The Reeb flow leaves the almost complex structure $J$ on $\mathcal{D}$ invariant.

(4) The Reeb flow leaves the $(1,1)$ tensor field $\Phi$ invariant.

(5) The contact metric structure $(M, \eta, g)$ is K-contact.

Proof. The conditions

$$
\eta(\xi)=1, \quad \xi\rfloor d \eta=0
$$


defining the Reeb vector field imply that both the symplectic form $d \eta$ and the contact form $\eta$ are invariant under the Reeb flow. From its definition Rei1] $g$ is bundle-like if and only if the transverse metric $g_{\mathcal{D}}$ is basic, that is, if and only if the Reeb flow leaves $g_{\mathcal{D}}$ invariant as well. Since $d \eta$ is invariant under the Reeb flow, $g_{\mathcal{D}}$ is invariant if and only if $J$ is invariant, or equivalently if and only if $\Phi$ is invariant.

Remark 2.6. We prefer the appellation bundle-like contact metric structure to the more common K-contact structure, since it is more descriptive and emphasizes the foliation aspect. We shall use these two terms interchangeably depending on the context. We also refer to the transverse structure $\left(d \eta, J, g_{\mathcal{D}}\right)$ on $M$ as a transverse almost Kähler structure.

There are obstructions to admitting K-contact metric structures. Indeed, it is well known [Bl] that K-contact metrics on a $2 n+1$ dimensional manifold can be characterized by the condition that the Ricci tensor in the direction of the Reeb vector field $\xi$ equals $2 n$. Thus, any metric of non-positive Ricci curvature cannot have a K-contact metric in its homothety class. However, it is much stronger to obtain obstructions which only depend on the smooth structure of the manifold. We mention one such result that follows directly from the work of Gromov $\mathrm{Gr}$, Carriere Car], and Inoue and Yano [IY].

Theorem 2.7. If a compact manifold $M$ admits a bundle-like contact metric structure, then the Gromov invariant $\|M\|$ and all the Pontrjagin numbers of $M$ vanish. In particular, if a compact manifold $M$ admits a decomposition as a connected sum $M=M_{1} \# \cdots \# M_{k}$, where for some $i=1, \cdots, k$ the manifold $M_{i}$ admits a metric of strictly negative sectional curvature, then $M$ does not admit any bundle-like contact metric structure.

\section{The Almost CR structure}

In this section we consider the integrability of the almost CR structure $J$. Let $(M, \eta, g)$ be a contact metric manifold. The almost CR structure $J$ is integrable, that is, $(\mathcal{D}, J)$ defines a $\mathrm{CR}$ structure on $M$ if and only if for any smooth sections $X, Y$ of $\mathcal{D}$ the following conditions hold:

(i) $[X, J Y]+[J X, Y]$ is a smooth section of $\mathcal{D}$.

(ii) $J[X, J Y]+J[J X, Y]=[J X, J Y]-[X, Y]$.

In our case condition (i) follows automatically from the antisymmetry of the symplectic form $d \eta$. Condition (ii) is the vanishing of the Nijenhuis tensor of $J$.

Now let $\nabla$ denote the Levi-Civita connection with respect to the metric $g$ on $M$. By restricting to $\mathcal{D}$ and taking the horizontal projection we get an induced connection $\nabla^{\mathcal{D}}$ on $\mathcal{D}$ defined by Ton]

$$
\nabla_{X}^{\mathcal{D}} Y= \begin{cases}\left(\nabla_{X} Y\right)^{h} & \text { if } X \text { is a smooth section of } \mathcal{D} \\ {[\xi, Y]^{h}} & \text { if } X=\xi\end{cases}
$$

where $Y$ is a smooth section of $\mathcal{D}$ and the superscript $h$ denotes the projection onto $\mathcal{D}$. An entirely standard computation gives

Proposition 3.2. Let $(M, \eta, g)$ be an contact metric manifold. Then $\nabla^{\mathcal{D}} J=0$ if and only if the almost CR-structure $J$ on $\mathcal{D}$ is integrable and $\mathcal{L}_{\xi} \Phi=0$. 
Proof. First we notice that as mentioned above condition (i) is automatically satisfied. Next, one easily sees that the invariance of $\Phi$ under $\xi$ holds if and only if $\nabla_{\xi}^{\mathcal{D}} J=0$. Now the connection $\nabla^{\mathcal{D}}$ is torsion-free [Ton, so

$$
\nabla_{X}^{\mathcal{D}} Y-\nabla_{Y}^{\mathcal{D}} X=[X, Y]^{h} .
$$

Now as above the vertical part of $N_{J}$ vanishes, and a straightforward computation gives

$$
N_{J}(X, Y)^{h}=\left(\nabla_{J X}^{\mathcal{D}} J\right)(Y)-\left(\nabla_{J Y}^{\mathcal{D}} J\right)(X)+J\left(\nabla_{Y}^{\mathcal{D}} J\right)(X)-J\left(\nabla_{X}^{\mathcal{D}} J\right)(Y) .
$$

The "only if" part clearly holds (which is all we shall need), and the "if" part follows by a standard computation (cf. [YK]).

Recall that a contact metric structure $(M, \eta, g)$ is said to be normal if the Nijenhuis tensor $N_{\Phi}$ defined by

$$
N_{\Phi}(X, Y)=[\Phi X, \Phi Y]+(\Phi)^{2}[X, Y]-\Phi[X, \Phi Y]-\Phi[\Phi X, Y]
$$

satisfies

$$
N_{\Phi}=-d \eta \otimes \xi .
$$

A normal contact metric structure on $M$ is also called a Sasakian structure.

Proposition 3.5. Let $(M, \eta, g)$ be a contact metric manifold. Then $(M, \eta, g)$ is normal (Sasakian) if and only if the almost CR structure $J$ is integrable and $\mathcal{L}_{\xi} \Phi=$ 0 .

Proof. For any vector fields $X, Y$ on $M$ we have

$$
\begin{aligned}
N_{\Phi}(X, Y)+d \eta(X, Y) \xi=[\Phi & X, \Phi Y]+(\Phi)^{2}[X, Y] \\
& -\Phi[X, \Phi Y]-\Phi[\Phi X, Y]+d \eta(X, Y) \xi .
\end{aligned}
$$

If $X$ and $Y$ are both horizontal, then this equals

$$
[\Phi X, \Phi Y]-[X, Y]-\Phi[\Phi X, Y]-\Phi[X, \Phi Y]
$$

whose vanishing is equivalent to (ii) above. Also applying $\eta$ to this equation and replacing $X$ by $\Phi X$ implies (i). If one vector field is vertical, say $X=\xi$, then we have

$$
\left.N_{\Phi}(\xi, Y)+d \eta(\xi, Y) \xi=(\xi\rfloor d \eta\right)(Y) \xi-\Phi \circ\left(\mathcal{L}_{\xi} \Phi\right)(Y) .
$$

So the result follows.

\section{The Leaf Closures of $\mathcal{F}_{\xi}$}

In this section we study the leaf closures of the characteristic foliation. In Mol1, Mol2 Molino has shown that on any compact connected manifold $M$ with a Riemannian foliation $\mathcal{F}$ there is a locally constant sheaf $C(M, \mathcal{F})$, called the commuting sheaf, consisting of germs of local transverse vector fields that are Killing vector fields with respect to the transverse metric, and whose orbits are precisely the closures of the leaves of $\mathcal{F}$. Moreover, Carrière Car] (see also the appendix in [Mol1] by Carrière) has shown in the case of Riemannian foliations of dimension one (Riemannian flows) that the leaf closures are diffeomorphic to tori, and that the flow is conjugate by the diffeomorphism to a linear flow on the torus.

Here we adapt this to our situation, that is, $(M, \eta, g)$ is a compact bundle-like metric contact manifold. We denote the isometry group of $(M, g)$ by $\Im(M, g)$, and 
the group of automorphisms of the K-contact structure $(M, \eta, g)$, by $\mathfrak{A}(M, \eta, g)$. When $M$ is compact the well known theorem of Myers and Steenrod says that $\mathfrak{I}(M, g)$ is a compact Lie group. Moreover, $\mathfrak{A}(M, \eta, g)$ is a closed Lie subgroup of $\mathfrak{I}(M, g)$ Tan1. In our case the Reeb flow belongs to the automorphism group $\mathfrak{A}(M, \eta, g)$ which is a compact Lie group. Thus, the closure $\mathfrak{T}$ of the Reeb flow is a compact commutative Lie group, i.e., a torus, which lies in $\mathfrak{A}(M, \eta, g)$. Now the Reeb flow is a strict contact transformation lying in the center of the group of strict contact transformations $[\mathrm{LM}$; hence, it lies in the center of $\mathfrak{A}(M, \eta, g)$. It follows that $\mathfrak{T}$ also lies in the center of $\mathfrak{A}(M, \eta, g)$. Summarizing we have

Proposition 4.1. Let $(M, \eta, g)$ be a compact bundle-like contact metric manifold. Then the leaf closures of the Reeb flow are the orbits of a torus $\mathfrak{T}$ lying in the center of the Lie group $\mathfrak{A}(M, \eta, g)$ of automorphisms of $(M, \eta, g)$, and the Reeb flow is the orbit of a linear flow on $\mathfrak{T}$.

The dimension of the torus in Proposition 4.1 is an invariant of the K-contact structure that we call the $\operatorname{rank}$ of $(M, \eta, g)$ and denote by $\operatorname{rk}(M, \eta)$. We have (see also [Ruk2])

Lemma 4.2. Let $(M, \eta, g)$ be a compact bundle-like contact metric manifold of dimension $2 n+1$. Then the $\operatorname{rank} \operatorname{rk}(M, \eta)$ depends only on the Pfaffian structure $(M, \eta)$ and satisfies $1 \leq \operatorname{rk}(M, \eta) \leq n+1$.

Proof. Consider the Lie algebra $\mathfrak{t}$ of $\mathfrak{T}$. It consists of the Reeb vector field $\xi$ together with the infinitesimal generators of the leaf closures. The projections of these generators onto $\mathcal{D}$ are global sections of Molino's commuting sheaf $C\left(M, \mathcal{F}_{\xi}\right)$. Thus, they give integral submanifolds of the subbundle $\mathcal{D}$. It is well known [LM] that the integral submanifolds of maximal dimension, that is, the Legendre submanifolds of the contact structure, have dimension $n$. Hence, these together with the Reeb vector field generate a torus of dimension at most $n+1$. Furthermore, Molino [Mol1] shows that the commuting sheaf is independent of the transverse metric, $\operatorname{so} \operatorname{rk}(M, \eta)$ is independent of $g$.

Now the $\operatorname{rank} \operatorname{rk}(M, \eta)$ is not an invariant of the contact structure $(M, \mathcal{D})$ but only of the Pfaffian structure $(M, \eta)$. The case $\operatorname{rk}(M, \eta)=1$ is the quasi-regular case, while the other extreme $\operatorname{rk}(M, \eta)=n+1$ is the toric case studied in [BM1, [BM2, BG3. Furthermore, Rukimbira Ruk1 showed that one can approximate any K-contact form $\eta$ by a sequence of quasi-regular K-contact forms in the same contact structure. Thus, every K-contact manifold has an $\eta$ of rank 1. Since we shall discuss this approximation in detail in the next section, we only mention here that one chooses a sequence of vector fields $\xi_{j}$ in $\mathfrak{t}$ with periodic orbits that converges to the Reeb vector field $\xi$. Then the dual 1 -forms $\eta_{j}$ are quasi-regular contact forms in the same contact structure.

\section{The proof of Theorem A}

We shall prove the following restatement of Theorem A:

Theorem $\mathbf{A}^{\prime}$. Let $(M, \eta, g)$ be a compact $K$-contact Einstein manifold. Then $(M, \eta, g)$ is Sasakian-Einstein.

Proof. We first prove the theorem under the assumption that $\eta$ is quasi-regular. By Thomas Tho and [BG1, $M$ is the total space of a principal $S^{1} \mathrm{~V}$-bundle over 
a compact almost Kähler orbifold $\mathcal{Z}$. Furthermore, by Bes] the induced metric $h$ on $\mathcal{Z}$ is almost Kähler-Einstein which has positive scalar curvature, since $g$ has positive scalar curvature. Now since Sekigawa's [Sek1], Sek2] proof of the Goldberg conjecture in the case of nonnegative scalar curvature only involves local curvature computations together with a Bochner type argument using Stokes Theorem, it carries over to the case of a compact orbifold. So the almost complex structure on $\mathcal{Z}$ is integrable, and $(\mathcal{Z}, h)$ is Kähler-Einstein. It then follows from the orbifold version of Hatakeyama [Hat that $(M, \eta, g)$ is normal, hence, Sasakian-Einstein. This proves the result under the assumption of quasi-regularity.

Now assume that $(M, \eta, g)$ is K-contact and Einstein, but not quasi-regular. Then by Proposition 4.1 the Reeb vector field $\xi$ lies in the commutative Lie subalgebra $\mathfrak{t}\left(M, \mathcal{F}_{\xi}\right) \subset \mathfrak{a}(M, g)$ which has dimension $k>1$. Thus, there exists a sequence of quasi-regular contact forms $\eta_{j}$ and Reeb vector fields $\xi_{j} \in \mathfrak{t}\left(M, \mathcal{F}_{\xi}\right)$ that approximate $(\eta, \xi)$ in the compact-open $C^{\infty}$ topology. (In what follows we use this topology on the space of smooth sections of all tensor bundles.) Explicitly, there is a monotonically decreasing sequence $\left\{\epsilon_{j}\right\}_{1}^{\infty}$ with $\lim _{j \rightarrow \infty} \epsilon_{j}=0$ such that

$$
\eta_{j}=f\left(\epsilon_{j}\right) \eta, \quad \xi_{j}=\xi+\rho_{j}, \quad f\left(\epsilon_{j}\right)=\frac{1}{1+\eta\left(\rho_{j}\right)},
$$

where $f\left(\epsilon_{j}\right)$ are positive functions in $C^{\infty}(M)$ that satisfy $\lim _{j \rightarrow \infty} f\left(\epsilon_{j}\right)=1$. Clearly $\rho_{j} \in \mathfrak{t}\left(M, \mathcal{F}_{\xi}\right)$ and $\lim _{j \rightarrow \infty} \rho_{j}=0$. Moreover, ker $\eta_{j}=$ ker $\eta=\mathcal{D}$, so we have the same underlying contact structure. We also have the following easily verified relations for the induced contact endomorphisms $\Phi_{j}$ :

$$
\Phi_{j}=\Phi-\frac{1}{1+\eta\left(\rho_{j}\right)} \Phi \rho_{j} \otimes \eta=\Phi-f\left(\epsilon_{j}\right) \Phi \rho_{j} \otimes \eta .
$$

This implies that $\Phi_{j} \xi_{j}=0$ and that the almost complex structure $J$ on $\mathcal{D}$ remains unchanged. However, the induced metrics become

$$
g_{j}=f\left(\epsilon_{j}\right) g_{\mathcal{D}} \oplus f\left(\epsilon_{j}\right)^{2} \eta \otimes \eta=g-\eta\left(\rho_{j}\right)\left(g_{\mathcal{D}}+2 \eta \otimes \eta\right)+o\left(\epsilon_{j}^{2}\right) .
$$

For $\epsilon_{j}$ small enough $g_{j}$ are well defined Riemannian metrics on $M$ which can easily be seen to satisfy the compatibility conditions

$$
g_{j}\left(\Phi_{j} X, \Phi_{j} Y\right)=g_{j}(X, Y)-\eta_{j}(X) \eta_{j}(Y) .
$$

Moreover, since $\xi_{j} \in \mathfrak{t} \subset \mathfrak{a}(M, \eta)$, it follows that the functions $f\left(\epsilon_{j}\right) \in C^{\infty}(M)^{\mathfrak{T}}$, where $C^{\infty}(M)^{\mathfrak{T}}$ denotes the subalgebra of $C^{\infty}(M)$ invariant under the action of the torus $\mathfrak{T}$. Thus, from (5.2) we have

$$
\mathcal{L}_{\xi_{j}} \Phi_{j}=0 .
$$

Hence $\left(M, \eta_{j}, \xi_{j}, \Phi_{j}, g_{j}\right)$ is a sequence of quasi-regular K-contact structures on $M$ whose limit with respect to the compact-open $C^{\infty}$ topology is the original K-contact Einstein structure $(M, \eta, \xi, \Phi, g)$. Now the metrics $g_{j}$ are not Einstein, but their Ricci tensor can be seen to satisfy

$$
\operatorname{Ric}_{g_{j}}=\lambda_{j} g_{j}+A\left(\epsilon_{j}, \rho_{j}, g\right),
$$

where $A\left(\epsilon_{j}, \rho_{j}, g\right)$ is a traceless symmetric 2-tensor field depending on $\epsilon_{j}, \rho_{j}, g$ that tends to 0 with $\epsilon_{j}$, and $\lambda_{j} \in C^{\infty}(M)$ satisfy $\lim _{j \rightarrow \infty} \lambda_{j}=2 n$.

Now there is a sequence of orbifold Riemannian submersions $\pi_{j}: M \longrightarrow \mathcal{Z}_{j}$, where $\left(\mathcal{Z}_{j}, h_{j}\right)$ are a sequence of compact almost Kähler orbifolds satisfying $\pi_{j}^{*} h_{j}=$ $f\left(\epsilon_{j}\right) g_{\mathcal{D}}$. Moreover, it follows from the above limits that the scalar curvatures of 
the $h_{j}$ are all positive. Notice that in Sekigawa's proof Sek2 of the positive scalar curvature Goldberg conjecture, the Einstein condition is not used until section 4 of [Sek2]. Following [Sek2] and making the necessary adjustments to our situation, we find that there are nonnegative numbers $\delta_{j}$ and nonnegative smooth functions $F_{j}$ such that

$$
\int_{\mathcal{Z}_{j}}\left(F_{j}+\frac{\check{s}_{j}}{n}\left\|\check{\nabla}_{j} \check{J}_{j}\right\|_{\mathcal{Z}_{j}}^{2}+\frac{1}{2 n}\left\|\check{\nabla}_{j} \check{J}_{j}\right\|_{\mathcal{Z}_{j}}^{4}\right) \sigma_{j} \leq \delta_{j},
$$

where $\check{\nabla}_{j}, \check{J}_{j}, \check{s}_{j}, \sigma_{j}$ and $\|\cdot\|_{\mathcal{Z}_{j}}$ are the Levi-Civita connection, almost complex structure, scalar curvature, volume element, and Riemannian norm, respectively on $\left(\mathcal{Z}_{j}, h_{j}\right)$. Now since the metrics $g, g_{j}$ are bundle-like the leaves of the characteristic foliation are geodesics and the O'Neil tensors $T$ and $N$ vanish Ton. Moreover, for any K-contact manifold of dimension $2 n+1$ the O'Neill tensor $A$ satisfies $\|A\|^{2}=$ $g(A \xi, A \xi)=2 n$. Thus, we have the relation between the functions $\lambda_{j}$ on $M$ and the scalar curvatures $\check{s}_{j}$ on $\mathcal{Z}_{j}$,

$$
\check{s}_{j}=(2 n+1) \lambda_{j}+2 n,
$$

so that $\lim _{j \rightarrow \infty} \check{s}_{j}=2 n+(2 n+1) \lim _{j \rightarrow \infty} \lambda_{j}=4 n(n+1)$. Furthermore, we have $\lim _{j \rightarrow \infty} \delta_{j}=0$. Thus, since $F_{j}$ (see [Sek2]) and $\check{s}_{j}$ are nonnegative for each $j$, the estimate (5.6) implies the estimate

$$
\left\|\check{\nabla}_{j} \check{J}_{j}\right\|_{\mathcal{Z}_{j}} \leq \delta_{j}^{\prime}
$$

where $\delta_{j}^{\prime}$ are nonnegative numbers satisfying $\lim _{j \rightarrow \infty} \delta_{j}^{\prime}=0$. Now for each $j$ the horizontal lift of $\check{\nabla} \check{J}_{j}$ is the horizontal projection $\left(\nabla_{j} J_{j}\right)^{h}=\left(\nabla_{j} \Phi_{j}\right)^{h}$, where $\nabla_{j}, J_{j}, \Phi_{j}$ are the corresponding Levi-Civita connection and tensor fields with respect to the metrics on $M$. But on $M J_{j}=J$ for all $j$ and we have

$$
\left\|(\nabla J)^{h}\right\|=\lim _{j \rightarrow \infty}\left\|\left(\nabla_{j} J\right)^{h}\right\|_{j} \leq \lim _{j \rightarrow \infty} \delta_{j}^{\prime}=0
$$

where $\|\cdot\|_{j}$ is the Riemannian norm with respect to $g_{j}$. So by Proposition 3.2 the almost $\mathrm{CR}$ structure on $\mathcal{D}$ is integrable which, by Proposition 3.5, implies that $(M, \eta, g)$ is Sasakian-Einstein.

Remark 5.10. Notice that by (5.7) the scalar curvatures $\check{s}_{j}$ of the orbifolds $\left(\mathcal{Z}_{j}, h_{j}\right)$ are close to $4 n(n+1)$. But the integrability argument actually holds for a much larger range of scalar curvatures, namely $\check{s}_{j} \geq 0$. We shall make use of this in section 7 when discussing $\eta$-Einstein metrics.

\section{Almost KäHLER CONES}

Here we give a corollary of Theorem A concerning almost Kähler cones. We consider the symplectification of $(M, \eta)$, namely, the symplectic cone

$$
C(M)=\left(M \times \mathbb{R}^{+}, d\left(r^{2} \eta\right)\right)
$$

We can extend the almost complex structure $J$ on $\mathcal{D}$ to an almost complex structure $I$ on $T C(M)$ by setting

$$
I=J \text { on } \mathcal{D}, \quad I \xi=-\Psi, \quad I \Psi=\xi,
$$


where $\Psi=r \frac{\partial}{\partial r}$ is the Euler vector field. Then the metric $g_{\mathcal{D}}+\eta \otimes \eta$ on $M$ corresponds to the metric $d r^{2}+r^{2}\left(g_{\mathcal{D}}+\eta \otimes \eta\right)$ on $C(M)$. We have arrived at:

Proposition 6.2. Contact metric geometry on $M$ corresponds to almost Kähler geometry on $C(M)$.

So what does the K-contact condition on $M$ correspond to? We have

Proposition 6.3. A compact metric contact manifold $\left(M, \eta, g_{M}\right)$ is K-contact if and only if $\left(C(M), d\left(r^{2} \eta\right), d r^{2}+r^{2} g_{M}\right)$ is almost Kähler with $\Psi-i \xi$ pseudo-holomorphic.

Proof. By the properties of the Reeb vector field one easily sees that $\Psi-i \xi$ is pseudoholomorphic, i.e., an infinitesimal automorphism of $I$ if and only if $\mathcal{L}_{\xi} J=0$. But this holds if and only if $\mathcal{L}_{\xi} g_{\mathcal{D}}=0$.

Remark 6.4. In the case that the complex vector field $\Psi-i \xi$ on $C(M)$ is not pseudo-holomorphic, a quotient formed by dividing by the resulting $\mathbb{C}^{*}$ action, or equivalently, by the symplectic reduction of the $S^{1}$ action, will lose both the almost complex structure and the Riemannian structure.

Corollary 6.5. Let $M$ be compact with a metric contact structure $\left(\eta, g_{M}\right)$, and consider the almost Kähler cone $\left(C(M), d\left(r^{2} \eta\right), d r^{2}+r^{2} g_{M}\right)$. Suppose that the $(1,0)$ vector field $\Psi-i \xi$ is pseudo-holomorphic and the cone metric $d r^{2}+r^{2} g_{M}$ is Ricci flat. Then the almost complex structure is integrable and the cone metric is CalabiYau.

Proof. It is well known that a cone metric $d r^{2}+r^{2} g_{M}$ on a cone $C(M)$ of dimension $N$ is Ricci flat if and only if the metric $g_{M}$ is Einstein with Einstein constant $N-2$. Thus, the result follows from Proposition 6.3 and Theorem A.

Another easy consequence of our results involves Vaisman's generalized Hopf manifolds. Consider an almost Kähler cone $\left(C(M), d r^{2}+r^{2} g_{M}\right)$. Then on $M \times S^{1}$ defined as the quotient manifold of $C(M)$ by the discrete group generated by $r \mapsto$ $e^{a} r$, where $0<a<1$ is fixed, the metric $g_{M}+\left(\frac{d r}{r}\right)^{2}$ is locally conformally almost Kähler. Furthermore, the vector field $\Psi$ and the almost complex structure $I$ pass to the quotient. Then we have

Corollary 6.6. Let $M$ be compact with a metric contact structure $\left(\eta, g_{M}\right)$ and consider the locally conformally almost Kähler manifold $\left(M \times S^{1}, g_{M}+\left(\frac{d r}{r}\right)^{2}\right)$. Suppose further that the $(1,0)$ vector field $\Psi-i \xi$ is pseudo-holomorphic and that the locally defined almost Kähler metrics $d r^{2}+r^{2} g_{M}$ are Ricci flat. Then the almost complex structure $I$ is integrable so the manifold $\left(M \times S^{1}, g_{M}+\left(\frac{d r}{r}\right)^{2}\right)$ is a locally conformal Calabi-Yau manifold [BG2].

\section{Some REMARKS ON $\eta$-EINSTEIN METRICS}

We conclude with some results about $\eta$-Einstein metrics. First recall [Tan2, YK]

Definition 7.1. A metric contact structure $(\eta, g)$ on $M$ is said to be $\eta$-Einstein if there are constants $a, b$ such that $\operatorname{Ric}_{g}=a g+b \eta \otimes \eta$.

Actually if $(M, \eta, g)$ is Sasakian and such a condition holds for $\operatorname{Ric}_{g}$, where $a, b$ are smooth functions, then these functions must be constant [YK]. In this section 
we shall prove:

Theorem 7.2. Let $(M, \eta, g)$ be a compact K-contact manifold such that $g$ is $\eta$ Einstein. Then

(i) If $a>-2$ the almost $C R$-structure $J$ is integrable, so $g$ is Sasakian. Moreover, for $\alpha=\frac{a+2}{2 n+2}$ the metric $\alpha g+\alpha(\alpha-1) \eta \otimes \eta$ is Sasakian-Einstein. Hence, $\pi_{1}(M)$ is finite.

(ii) If $a=-2$ the almost $C R$-structure is integrable, so $(g, \eta)$ is Sasakian $\eta$ Einstein. Moreover, if $M$ has finite fundamental group, then $\operatorname{rk}(M, \eta)=1$ so the K-contact manifold $(M, \eta, g)$ is quasi-regular, and the total space of a principal $S^{1}$ V-bundle over a Calabi-Yau orbifold.

(iii) If $a<-2$, then $\operatorname{rk}(M, \eta)=1$ so the $K$-contact manifold is quasi-regular, and the total space of a principal $S^{1}$ V-bundle over an almost Kähler-Einstein orbifold with Einstein constant $2 n(a+2)$.

Proof. (i) Notice that Tanno [Tan2 proves the second statement of (i) under the assumption that $(M, \eta, g)$ is Sasakian. However, as we shall see, this assumption is not necessary. Since the O'Neill tensors $T$ and $N$ vanish and $A$ satisfies $g\left(A_{X}, A_{Y}\right)=g(\Phi X, \Phi Y)=g(X, Y)$, it follows that the Ricci curvature of the transverse metric $g_{\mathcal{D}}$ satisfies

$$
\operatorname{Ric}_{g_{\mathcal{D}}}=\left.\operatorname{Ric}_{g}\right|_{\mathcal{D} \times \mathcal{D}}+\left.2 g\right|_{\mathcal{D} \times \mathcal{D}}
$$

The condition that on $\mathcal{D}$ the Ricci curvature satisfies $\operatorname{Ric}_{g}>-2$ is equivalent to the condition that $\operatorname{Ric}_{g_{\mathcal{D}}}>0$. Now even though in general we do not have a Riemannian submersion (even in the orbifold sense), the canonical variation described in Besse Bes applies equally well to our foliation since it is based on the O'Neill formulas which do hold in our case. Then one easily sees that by choosing $\alpha=\frac{a+2}{2 n+2}$ the metric $g^{\prime}=\alpha g+\alpha(\alpha-1) \eta \otimes \eta$ is Einstein, so $\left(M, \alpha \eta, g^{\prime}\right)$ is K-contact and Einstein. Thus, by Theorem A it is Sasakian-Einstein. Since the underlying almost CRstructure hasn't changed, the original $\mathrm{K}$-contact structure $(M, \eta, g)$ is Sasakian; hence, it is Sasakian $\eta$-Einstein.

(iii) Let $a<-2$. Then from (7.3) $\operatorname{Ric}_{g_{\mathcal{D}}}<0$. Suppose that the K-contact structure $(M, \eta, g)$ is not quasi-regular. Then Molino's commuting sheaf $C\left(M, \mathcal{F}_{\xi}\right)$ is non-vanishing. So by a perturbation of the K-contact structure there is a quasiregular K-contact structure $\left(\eta^{\prime}, g^{\prime}\right)$ with the same commuting sheaf. But by a theorem of Molino and Sergiescu [MoSe] the sheaf $C\left(M, \mathcal{F}_{\xi}\right)$ has a global trivialization. Thus, there are transverse Killing vector fields on $\left(M, \eta^{\prime}, g^{\prime}\right)$. These project to non-trivial Killing vector fields on a compact orbifold $\mathcal{Z}^{\prime}$ with negative Ricci curvature. But as in the manifold case a compact orbifold with negative Ricci curvature can have no Killing fields. This gives a contradiction.

(ii) By (7.3) the case $a=-2$ corresponds to the vanishing of the scalar curvature of the transverse metric $g_{\mathcal{D}}$. But then as mentioned in Remark 5.10 the proof of Theorem A holds in this case, and the almost CR-structure $J$ is integrable. In this case the transverse geometry is Calabi-Yau, and the metric $g$ is Sasakian $\eta$ Einstein with $a=-2$ and $b=2 n+2$. To prove the second statement we proceed as in the proof of (iii), only now the transverse Ricci tensor vanishes implying that any Killing fields on $\mathcal{Z}^{\prime}$ must be parallel. Moreover, the vector space of these Killing fields has dimension equal to the first Betti number $b_{1}\left(\mathcal{Z}^{\prime}\right)$. But the finiteness of $\pi_{1}(M)$ together with the long exact homotopy sequence of the orbifold fibration 
$\pi: M \longrightarrow \mathcal{Z}^{\prime}$ implies that $\pi_{1}^{\text {orb }}\left(\mathcal{Z}^{\prime}\right)$ is also finite, and this implies that $H_{1}\left(\mathcal{Z}^{\prime}, \mathbb{Q}\right)=$ $H_{1}^{\text {orb }}\left(\mathcal{Z}^{\prime}, \mathbb{Q}\right)=0$. This gives a contradiction.

Actually our proof gives a bit more:

Proposition 7.4. Let $(M, \eta, g)$ be a compact K-contact manifold. Suppose that the transverse Ricci tensor satisfies $\operatorname{Ric}_{g_{\mathcal{D}}} \leq 0$ and $\operatorname{Ric}_{g_{\mathcal{D}}}(v, v)<0$ for all $v \in \mathcal{D}_{p}-\{0\}$ for some $p \in M$. Then $\operatorname{rk}(M, \eta)=1$ so the $K$-contact manifold is quasi-regular, and the total space of a principal $S^{1} V$-bundle over an almost Kähler orbifold.

\section{A remark on contact 3-Structures}

It is interesting to inquire about a quaternionic analogue of our main theorem. In this regard it has been recently observed by Kashiwada Kas that a much stronger result is available. Indeed, a metric contact 3-structure on a manifold $M$ is a triple of contact structures $\left\{\eta^{a}, \xi^{a}, \Phi^{a}\right\}_{a=1}^{3}$ associated with the same metric $g$ such that

$$
\Phi^{a} \circ \Phi^{b}-\xi^{a} \otimes \eta^{b}=-\epsilon^{a b c} \Phi^{c}-\delta^{a b} \mathrm{id}
$$

where $\epsilon^{a b c}$ is the totally antisymmetric symbol and sum over repeated indices is used. If each contact structure is normal the triple is called 3-Sasakian (cf. BG1).

Theorem (Kashiwada). Every metric contact 3-structure is 3-Sasakian.

The key to the proof of this theorem is a result of Hitchin buried deep in his famous stable pairs paper [Hit]. This result says that an almost hyperkähler structure must be hyperkähler. More explicitly, if one has a manifold with a triple of almost complex structures satisfying the algebra of the quaternions, together with a triple of compatible Kähler forms all of which are closed, then the almost complex structures are integrable. That is, the quaternionic algebra and the closedness of the forms are strong enough to force integrability. Then Kashiwada's Theorem follows from Hitchin's Lemma together with the following quaternionic analogue of Proposition 6.2:

Proposition 8.1. A Riemannian manifold $(M, g)$ has a compatible contact 3structure $\left(\eta^{a}, \xi^{a}, \Phi^{a}\right)$ if and only if the cone $\left(C(M), d r^{2}+r^{2} g\right)$ is almost hyperkähler. Furthermore, $(M, g)$ is 3-Sasakian if and only if $\left(C(M), d r^{2}+r^{2} g\right)$ is hyperkähler.

We conclude by mentioning that some weaker results have been obtained in the last few years, first by Tanno Tan3] and then by Jelonek [Jel]. In 1996 Tanno observed that in dimension 7 any 3 -K-contact manifold must be 3-Sasakian and later Jelonek (using similar techniques but assuming leaf compactness of the associated 3dimensional foliation) extended this result to any 3-Sasakian dimension other than 11. Neither of the two authors noticed Hitchin's result. Instead they considered geometry of the associated foliation of $M$ by 3-dimensional leaves. It is likely that one could also give a direct proof of Kashiwada's Theorem working exclusively on $M$.

\section{ACKNOWLEDGMENTS}

Both authors would like to thank the Erwin Schrödinger International Institute for Mathematical Physics in Vienna for support and hospitality, where this work began. 


\section{REFERENCES}

[BM1] A. Banyaga and P. Molino, Géométrie des formes de contact complètement integrable de type torique, Séminaire Gaston Darboux, Montpelier, 1991-1992, 1-25. MR 94e:53029

[BM2] A. Banyaga and P. Molino, Complete Integrability in Contact Geometry, Pennsylvania State University preprint PM 197, 1996.

[Bes] A. L. Besse, Einstein Manifolds, Springer-Verlag, New York (1987). MR 88f:53087

[BG1] C. P. Boyer and K. Galicki, 3-Sasakian Manifolds, Surveys in Differential Geometry, VI; Essays on Einstein Manifolds, International Press 1999; C. LeBrun and M. Wang, Eds., pp. 123-184.

[BG2] C. P. Boyer and K. Galicki, On Sasakian-Einstein Geometry, preprint DG/981108, to appear in Int. J. Math.

[BG3] C. P. Boyer and K. Galicki, A Note on Toric Contact Geometry, The Journal of Geometry and Physics 35 (2000), 288-298.

[Bl] D. E. Blair, Contact Manifolds in Riemannian Geometry, Lecture Notes in Mathematics 509, Springer-Verlag, New York 1976. MR 57:7444

[BW] W.M. Boothby and H.C. Wang, On Contact Manifolds, Ann. of Math. 68 (1958), 721-734. MR 22:3015

[Car] Y. Carrière, Les propriétés topologiques des flots riemanniens retrouvées à l'aide du théorème des variétés presque plates, Math. Z. 186 (1984), 393-400. MR 86f:53048

[Gol] S.I. Goldberg, Integrability of almost Kaehler manifolds, Proc. Amer. Math. Soc. 21 (1969), 96-100. MR 38:6514

[Gr] M. Gromov, Volume and bounded cohomology, Inst. Hautes Études Sci. Publ. Math. 56 (1982), 213-307. MR 84h:53053

[Hat] Y. Hatakeyama, Some notes on differentiable manifolds with almost contact structures, Tôhuku Math. J. 15 (1963), 176-181. MR 27:705

[Hit] N.J. Hitchin, The self-duality equations on a Riemann surface, Proc. London Math. Soc. 55 (1987), 59-126. MR 89a:32021

[IY] H. Inoue and K. Yano, The Gromov invariant of negatively curved manifolds, Topology 21 (1981), 83-89. MR 82k:53091

[Jel] W. Jelonek, Positive and negative 3-K-contact structures, Proc. Amer. Math. Soc. (to appear).

[Kas] T. Kashiwada, On a contact 3-structure, preprint.

[LM] P. Libermann and C.-M. Marle, Symplectic Geometry and Analytical Mechanics, D. Reidel Publishing Co., Dordrecht, 1987. MR 88c:58016

[Mol1] P. Molino, Riemannian Foliations, Progress in Mathematics 73, Birkhäuser, Boston, 1988. MR 89b:53054

[Mol2] P. Molino, Feuilletages riemanniens sur les variétés compactes; champs de Killing transverses, C.R. Ac. Sci. Paris Sér. A 289 (1979), 421-423. MR 80j:57026

[MoSe] P. Molino and Vlad Sergiescu, Deux remarques sur les flots riemanniens, Manus. Math. 51 (1985), 145-161. MR 86h:53035

[Rei1] B.L. Reinhart, Foliated manifolds with bundle-like metrics, Ann. of Math. 69 (1959), 119-132. MR 21:6004

[Rei2] B.L. Reinhart, Differential Geometry of Foliations, Springer-Verlag, New York, 1983. MR 85i:53038

[Ruk1] P. Rukimbira, Chern-Hamilton's conjecture and K-contactness, Hous. J. of Math. 21 (1995), 709-718. MR 96m:53032

[Ruk2] P. Rukimbira, The dimension of leaf closures of K-contact flows, Ann. Global Anal. and Geom. 12 (1994), 103-108. MR 95f:53072

[Sek1] K. Sekigawa, On some 4-dimensional compact Einstein almost Kähler manifolds, Math. Ann. 271 (1985), 333-337. MR 86g:53054

[Sek2] K. Sekigawa, On some compact Einstein almost Kähler manifolds, J. Math. Soc. Japan 39 (1987), 677-684. MR 88j:53047

[Tan1] S. Tanno, The automorphism groups of almost contact Riemannian manifolds, Tôhoku Math. J. 21 (1969), 21-38. MR 39:3428

[Tan2] S. Tanno, Geodesic flows on $C_{L}$-manifolds and Einstein metrics on $S^{3} \times S^{2}$, in Minimal submanifolds and geodesics (Proc. Japan-United States Sem., Tokyo, 1977), pp. 283-292, North Holland, Amsterdam-New York, 1979. MR 81g:58027 
[Tan3] S. Tanno, Remarks on a Triple of K-contact Structures, Tôhoku Math. J. 48 (1996), 519-531. MR 97i:53055

[Tho] C.B. Thomas, Almost regular contact manifolds, J. Differential Geom. 11 (1976), 521-533. MR 57:4195|

[Ton] P. Tondeur, Geometry of Foliations, Birkhäuser, Boston, 1997. MR 98d:53037

[YK] K. Yano and M. Kon, Structures on Manifolds, Series in Pure Mathematics 3, World Scientific Pub. Co., Singapore, 1984. MR 86g:53001

Department of Mathematics and Statistics, University of New Mexico, Albuquerque, New MeXico 87131

E-mail address: cboyer@math.unm.edu

URL: http://www.math.unm.edu/ cboyer

Department of Mathematics and Statistics, University of New Mexico, Albuquerque, New Mexico 87131

E-mail address: galicki@math.unm.edu

URL: http://www. math.unm.edu/ ${ }^{\text {galicki }}$ 